\title{
An Adaptive Multiresolution Method for Speckle Noise Reduction in Synthetic Aperture Radar Images
}

\author{
Ana Vidal-Pantaleoni,David Martí, Miguel Ferrando \\ Departamento de Comunicaciones, Universidad Politécnica de Valencia \\ Camino de Vera s/n, E-46071 Valencia, Spain \\ Tel.: +34-963879710, Fax: +34-963877309, E-mail: avidal@dcom.upv.es
}

Abstract -- This paper introduces a method for Speekle noise reduction based on recent multiresolution algorithms and a new adaptive approach. It combines the characteristics for additive noise reduction of soft thresholding with the localization of information in the coarsest levels of the wavelet transform, being its main contribution to adapt the effective threshold to the level of resolution in the wavelet transform and to the local features of the image. The method has been applied successfully to the elimination of speckle noise of simulated SAR images. It has been applied to an optical image corrupted with multiplicative noise that simulates SAR statistics and it has been compared to other well-known noise reduction methods.

\section{INTRODUCTION}

Speckle noise is a well-known distorting effect that is present in Synthetic Aperture Radar (SAR) images. The high coherence of the emitted signal causes a pattern of interference due to the roughness of the surface. Noise affects severely post-processing applications like segmentation-and classification. For that reason, it is very important to reduce this effect before extracting scene features [1].

Many methods have been already developed to improve image quality by preserving scene texture and features. Some of them are based on statistical locally adapted filters and recently, methods based on multiresolution decomposition have appeared in the literature $[2,3,4,7]$. In this paper, a method based on Wavelet decomposition with a new adaptive approach is presented. It combines the characteristics of additive noise reduction of soft thresholding with the localization of information in the coarsest levels of the Wavelet transform, being its main contribution to adapt the effective threshold to the level of decomposition in the Wavelet Transform.

\section{BASIC THEORY}

Speckle contribution to the SAR image is often considered as noise, although it is a deterministic phenomenon produced by the eoherent contributions of the terrain scattering points. That is due to the high complexity of the interference mechanism in the Speckle formation process as described in [1]. When the SAR complex signal is considered as an independent stochastic process with exponential distribution and two degrees of freedom; then the intensity of the SAR image is Rayleigh distributed [1,2]. That approximation gives an accurate model but it is assumed that each resolution cell is formed by a large number of scattering points and the return signal can be regarded as a random walk in the complex domain. In the following, Speckle noise is supposed to be dependent on the signal intensity in the sense that noise level increases with the brightness. Therefore, a simple statistical model based on multiplicative noise is assumed as described in the literature $[1,4,7]$.

SAR Speckle noise reduction can be performed by several means. One of them is the multilook operation. It obtains an average of several images taken with a different antenna subaperture. In that process, each of the sub-apertures or spectral windows loses resolution compared to the original. One second approach is to process the original SAR image preserving the spatial resolution. In this work, information processing has been carried out in the Wavelet domain.

Wavelet Transform [3,4] performs a hierarchical decomposition of the signal space into a nested sequence of approximation spaces. The process creates a pyramid of lower resolution approximations. The direct wavelet transform of the signal $x(t)$ can be expressed as:

$$
\begin{gathered}
x_{n}^{m}=\int_{-\infty}^{\infty} x(t) \Psi_{n}^{m}(t) d t \\
\Psi_{n}^{m}(t)=2^{m / 2} \Psi\left(2^{m}-n\right)
\end{gathered}
$$

where $\Psi_{n}^{m}(t)$ represents a set of basis functions that are dilations and translations of a single function $\Psi(t)$ called mother wavelet. The inverse discrete wavelet transform gives the signal reconstruction:

$$
x(t)=\sum_{n} \sum_{m} x_{n}^{m} \Psi_{n}^{m}(t)
$$

The Wavelet decomposition in our case is a two dimensional problem and the implementation of the transformation has been approached through the efficient tree decomposition described by Mallat in [3]. The original image is progressively decomposed by filtering and decimation. The Wavelet decomposition has an interesting property of simultaneous space and frequency localization and it eases the reduction of noise.

\section{ALGORITHM DESCRIPTION}

The Speckle reduction method is structured in several stages. The approach described in [7] has been followed with some new features. First, a logarithmic operator is applied to the SAR image as described in [4]. Then, it is decomposed using a multiresolution representation and the signal is 
processed in that domain. Finally, an inverse Wavelet transformation and inverse logarithm operator are applied.

Since the assumption of a multiplicative noise model is adopted, then the logarithm transformation is applied in order to separate the noise contribution in an additive manner as described in $[1,7]$. Therefore, the noise dependence on the signal is eliminated. Obviously, the logarithm transformation is not a linear operator, but it has been shown that it does not affect severely the following multiresolution decomposition. There are other techniques for obtaining signal independent noise like normalization $[4,5]$. It has been also tested, but logarithm transformation has been selected because it has given the best results concerning noise reduction.

Then, the Wavelet Transform is used to obtain the multiresolution decomposition. The selected decomposition has been a three level pyramid using Daubechies family of $16^{\text {th }}$ order as described in [6]. This is a widely used decomposition that obtains compactly supported orthonormal basis decomposition.

The innovation to the soft thresholding method described in [7] is that coefficient processing is carried out independently in each detail sub-image. As a consequence, thresholding is applied to each coefficient following:

$$
\begin{gathered}
w^{\prime}=\operatorname{sign}(w) \cdot(|w|-T), \\
T=T_{e f} \sigma,
\end{gathered}
$$

where the threshold $T$ is obtained from the effective threshold $T_{e f}$ that is a design parameter and the standard deviation $\sigma$ of the processed coefficients. Soft-thresholding is a sub-optimal process, so an adequate threshold choice is a critical step. When the value is too high, the image gets distorted. On the contrary, when $T_{\text {ef }}$ is too small, noise cancellation efficiency is reduced drastically.

In the proposed method, an adaptive threshold is used in two steps. First, only the sub-image coefficients are taken into consideration for the computation of the standard deviation of the sub-image. It means that the threshold is adjusted to each sub-image characteristics. In a second stage, the threshold depends on the level of resolution according to the following equation that is a modified version of (5)

$$
T_{n m}=\gamma^{(n-1)} T_{e f} \sigma, \quad \mathrm{n}=1, \ldots, \mathrm{N}
$$

where $n$ is the decomposition level, $m$ is a sub-image index and $\gamma$ is the SNR adjustment parameter. When $n$ equals zero, no thresholding is applied because the low pass smooth subimage retains most of the information and distortion effects would be more severe than possible noise cancellation. The SNR of the transformed image decreases as the level of the decomposition $n$ increases. Meanwhile, the threshold is adapted locally to the resolution level. In summary, this adaptive soft-thresholding noise reduction gets an adjustment not only to the particular statistics of the sub-image (standard deviation) but also to the SNR of the resolution level.

The last stages are inverse Wavelet transformation and exponential operation in order to acquire the final SAR image with an improvement in its radiometric quality.

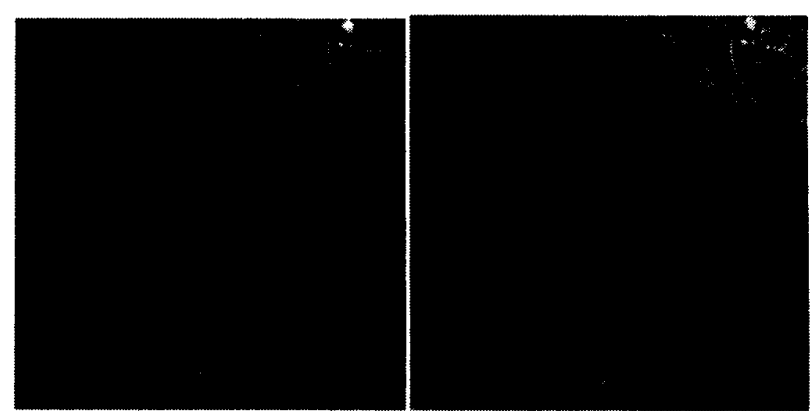

Fig. 1. Original and noisy aerial image

\section{RESULTS}

A simulation has been carried out using an optical aerial image corrupted with multiplicative noise. The original and the noisy image with a SNR of $14.7 \mathrm{~dB}$ are shown in Fig. 1. The selected image presents some detail features like roads and fields and different textures, that are blurred when the noise model is applied to the signal.

In the following results, the performance of the different algorithms is exhaustively analyzed. The evaluation has been done varying the values of the effective soft threshold $T_{e f}$ and the parameter $\gamma$ that is adjusted according to the image SNR. The results are presented in terms of cancelled mean square error that is equivalent to noise reduction. Therefore, the higher the magnitude of the graph, the best performance is achieved. The noise reduction can be measured globally, but no information about edge and detail preservation would be provided. For that reason, a mask has been designed and used to classify the error cancellation over edges or uniform regions. The mask is provided by a Sobel edge detector and a low pass filter used to smooth the result. So, a normalized edge mask is obtained.

The results of the proposed adaptive soft thresholding scheme are shown in Fig. 2. It can be observed that the method gets an optimum noise cancellation when the effective threshold is equal to 0.1 and $\gamma$ is equal to 3.75 .

The evaluation of the adaptive soft-threshold technique in comparison with other methods is shown in Fig. 3. It shows the edge error cancellation in relation to the achieved uniform error cancellation. These graphs show a maximum value that should be the ideal choice for the parameter selection. The implemented methods are the Wiener filter, soft-thresholding as described by Donoho in [7] and the adaptive version of soft-thresholding. The best result is clearly obtained for the proposed adaptive technique and one possible reason for such result is that more information about the image is considered in the noise reduction process.

The reconstructed image with processing parameters of an effective threshold equal to 0.1 and $\gamma$ equal to 3.75 is shown in Fig. 4. As it can be observed, noise is decreased in the uniform areas, but the detail is preserved at the edges. 


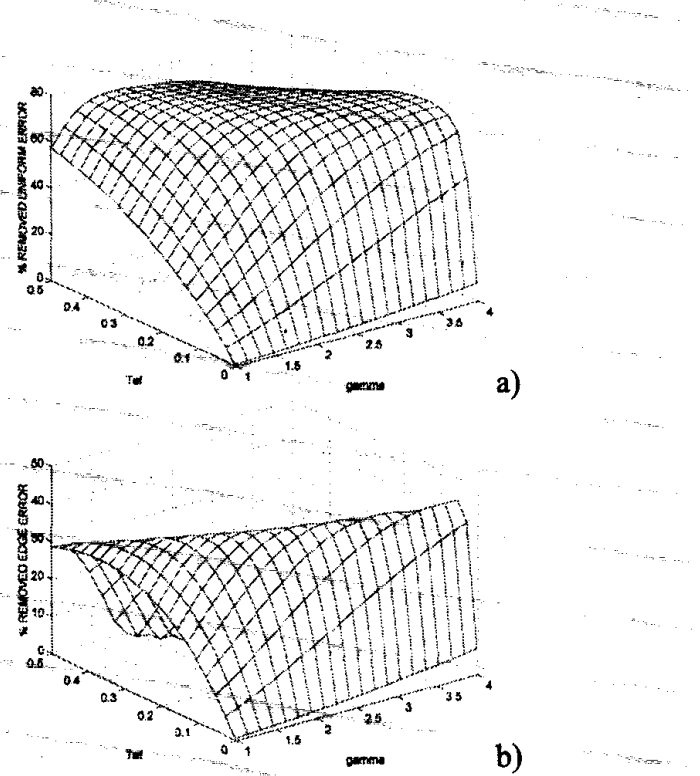

Fig. 2. Cancelled error in: a) uniform areas; b) edges

\section{CONCLUSIONS}

A study on different SAR de-speckling techniques has been described. The basic assumption has been to consider a multiplicative noise model for the SAR image.- A test environment has been created for comparing the performance of different Speckle reduction methods and the analysis has taken into account edge preservation through the classification of edge error and uniform error. The new version of the soft-thresholding has provided the best results at low computational cost: It provides the best performance in edge areas as well as uniform regions. Although it cancels a higher amount of noise, it does not introduce detail distortion. Soft thresholding of the Wavelet coefficients is implemented with a threshold that depends on the level of decomposition. Therefore, a double adaptation process is obtained depending on the characteristics of each subband. This process cancels a high amount of speckle noise, preserving the detail that is localized in the space domain thanks to the Wavelet transformation.

The new method provides speckle noise reduction and better results concerning detail distortion than the original soft thresholding technique. Furthermore, the computational effort of this method is not high compared to the other standard techniques. The total error with respect to the original optical image can be measured and it has been classified through a mask in two categories: uniform region error and edge error. This tool gives valuable information for algorithm assessment. Adaptive soft thresholding has outperformed the other methods in both quality and efficiency. So, it has paved the way for the development of further experiments including the test over other SAR ERS images with different structure and textures.

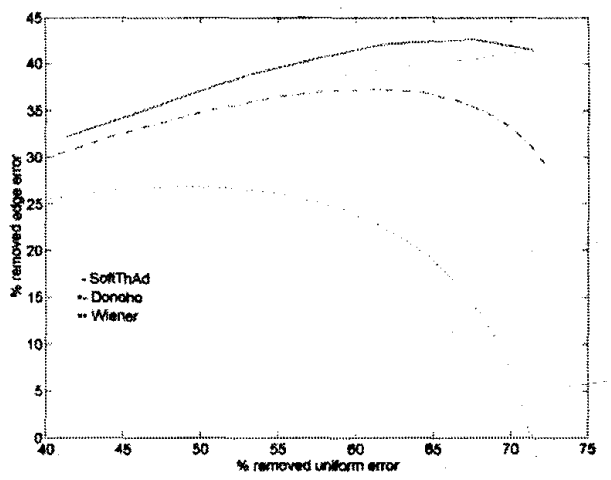

Fig. 3. Comparison of noise reduction methods

\section{REFERENCES}

[1] J. C. Dainty, Laser Speckle and Related Phenomena. Second Enlarged Edition, 1984.

[2] M. Datcu, D. Luca and K. Seidel, "Multiresolution Analysis of SAR Images". In Proc of EUSAR'96, Königswinter, Germany 1996.

[3] S. G. Mallat, "A Theory for Multiresolution Signal Decomposition", IEEE Trans. on Pattern Anal. Machine Intell., vol. 11, pp. 674-693, 1989.

[4] M. Simard and G. DeGrandi, "Analysis of Speckle Noise Contribution on Wavelet Decomposition of SAR Images", IEEE Trans. on Geosci. and Remote Sensing, vol. 36 , no. 6, pp. 1953-1962, 1998.

[5] M. Simard, "Extraction of Information and Speckle Noise Reduction in SAR Images Using the Wavelet Transform", in Proc. of the IGARSS'98, vol. 1, pp. 4-6, 1998.

[6] I. Daubechies, "Orthonormal Bases of Compactly Supported Wavelets". Comm. Pure Appl. Math., vol. 441, pp. 909-996, 1988.

[7] D. L. Donoho, "De-noising by Soft Thresholding". IEEE Trans. on Information Theory, vol. 41, pp: 613-627, 1995.

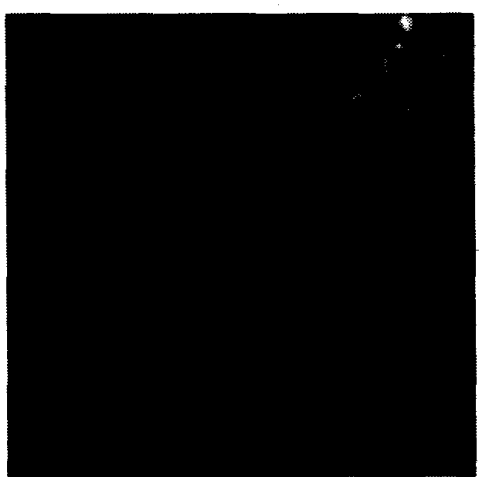

Fig. 4. Reconstructed image 